\title{
Offloading the diabetic foot: toward healing wounds and extending ulcer-free days in remission
}

\author{
Jano A Boghossian' \\ John D Miller ${ }^{2}$ \\ David G Armstrong' \\ 'Department of Surgery, Southern \\ Arizona Limb Salvage Alliance, \\ University of Arizona, Tucson, AZ, \\ ${ }^{2}$ Department of Podiatric Surgery and \\ Medicine, DVA Maryland Healthcare \\ System, Baltimore, MD, USA
}

This article was published in the following Dove Press journal:

Chronic Wound Care Management and Research

14 July 2017

Number of times this article has been viewed

\begin{abstract}
Management of the diabetic foot is multifaceted and requires constant monitoring from patients and health care providers. The alarmingly high rate of recurrence of ulcerations in diabetic foot requires a change in our approach to care and to the vernacular in the medical literature. With its high rates of morbidity and recurrence, care of the complex diabetic foot may be aptly comparable to many forms of cancer. Therefore, our efforts should be not only in rapid healing of open wounds but also in maximizing ulcer-free days for the patient in diabetic foot remission. One facet of the multidisciplinary approach in managing wounds is achieved by reducing peak plantar pressures by offloading the foot with various conservative and surgical techniques aimed at reducing areas of stress caused by ambulation and improper shoe gear. Evidence supports the use of total contact casts as the gold standard for offloading open wounds; however, other methods have gained popularity as well. Novel approaches in surgical techniques and advances in wearable technology appear to show promise in measuring and modulating dangerous pressure and inflammation to extend remission and improve quality of life for these most complex patients.
\end{abstract}

Keywords: diabetic foot, ulcer, remission, amputation, offloading, wearables

Diabetes and other noncommunicable diseases of decay are now the leading cause of global mortality in the developed and developing world. ${ }^{1}$ As the continuously growing epidemic of diabetes further expands, it inflicts affected patients with a myriad of subsequent diabetic complications and comorbidities that necessitate further financial investments in managing these patients in a multidisciplinary setting to save limbs from amputation. There are several well-known guidelines for prolonging remission from ulceration in the diabetic foot with the mind-set of "active prevention", including having a heightened awareness of these risk factors associated with ulceration, patient education, staying active, frequent inspection of the feet, reducing friction by wearing diabetic socks and proper footwear, avoiding self-injurious behavior such as walking barefoot, and long-term management of the various comorbid systemic diseases associated with diabetes. ${ }^{2,3}$

Due to the economic, physical, and psychological cost of diabetic foot ulcers (DFUs), efforts to delay or prevent their development are a prudent use of medical resources. Remarkably, people with DFUs have a risk of hospitalization that is comparable to or exceeding heart failure, pulmonary disease, renal disease, and most cancers. ${ }^{4}$ Patients with diabetes mellitus (DM) undergoing treatment of DFUs often present with confounding factors and subsequently experience high rates of recurrence. Of the individuals who successfully heal a DFU, the risk of ulcer recurrence within
Correspondence: David G Armstrong Southern Arizona Limb Salvage Alliance, University of Arizona, I50I N Campbell Ave, Bldg 20I, Rm 4402, Tucson, AZ 85724, USA

Tel +I 520626 I349

Email armstrong@usa.net 
the first year of healing a prior ulceration is likely $40 \% .^{5,6}$ Naturally, these devastating rates of recurrence lend themselves to comparison with rates of the deadliest of cancers and warrant a change in terminology and format of communicating and counseling this patient population. ${ }^{7}$ We believe a change in the syntax of resolved DFUs from the past tense of "healed" to the active "in remission" best represents the threat to the patient and the need for ongoing preventative care. This has the added benefit of preventing patients from being lost to follow-up or prudent at-home care as patients are now tasked with increasing their own "ulcer-free days" and reducing the severity of recurrences rather than simply "remaining healed". ${ }^{6-8}$

\section{Reducing peak plantar pressures The use of custom footwear for offloading}

In treating DFUs, alleviating pressure at areas of high vertical and shear stress can be achieved by offloading the foot with various techniques, starting with custom therapeutic footwear. Patients with diabetes have higher peak plantar pressures (Figure 1); ${ }^{9}$ coupled with repetitive pedal stress caused by osseous and structural abnormalities of the foot in the presence of neuropathy and peripheral arterial disease, these risk factors are some of the strongest predictors of ulcer development. ${ }^{10}$ As a result, redistribution of constant plantar pressure is of utmost importance in managing a diabetic in remission. ${ }^{11}$ In quantifying an in-shoe peak plantar pressure threshold, $200 \mathrm{kPa}$ has been suggested as a meaningful value below which ulceration may be prevented, as seen from patients whose previous ulceration sites have remained in remission. This number, of course, is a compromise, as other ingredients (shear stress, duration of standing, and cycles of repetitive stress) may also increase the risk for those with lower peak plantar stress numbers. ${ }^{12,13}$

In a 2008 study, shoe modification was more commonly used as a method of offloading compared with the gold

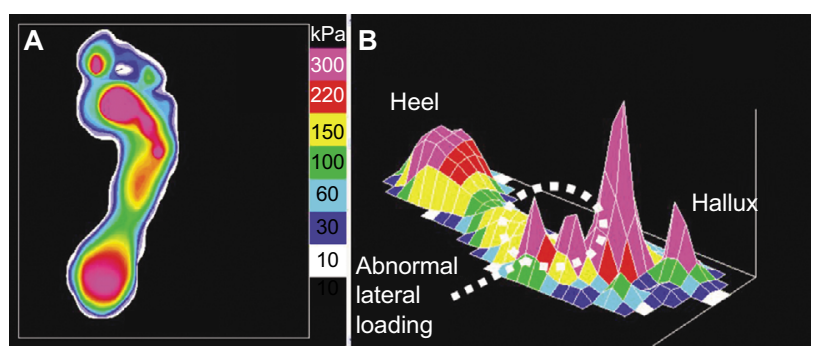

Figure I Peak pressure profile for a diabetic foot.

Notes: (A) Two-dimensional (2D) depiction of plantar pressure and (B) 3D depiction of plantar pressure (note the abnormal spikes along the lateral forefoot). standard of offloading: the total contact cast (TCC).${ }^{14}$ Custom footwear such as diabetic shoes and inserts have been shown to be effective at reducing peak plantar pressures and shear forces to reduce ulcer recurrence. ${ }^{3} \mathrm{~A}$ randomized control trial demonstrated that silicone orthoses can significantly reduce the incidence of a primary ulcer in those at risk with preulcerative lesions, ${ }^{3}$ and one study showed a $30 \%$ difference in ulcer recurrence for patients who wore diabetic therapeutic shoes in comparison to those who wore normal shoe gear. ${ }^{15}$

Although most physicians understand the tenets of diabetic ulcer management, the cost-benefit analysis associated with the real-life application of the gold standard TCC might influence specialists to resort to less optimal offloading techniques. Little harm has been reported in the literature from properly fitting custom footwear, and the benefits likely outweigh the potential harm, although patient adherence to these therapies is one of the largest hurdles clinicians must overcome in dealing with ulcer recurrence.

\section{Casting methods for offloading}

Medical literature supports the use of TCCs as the gold standard treatment for offloading high peak plantar pressure for patients with active neuropathic ulcerations. ${ }^{16}$ Systematic reviews indicate that the use of nonremovable casting devices like TCCs significantly improve healing of neuropathic plantar forefoot ulcers compared with removable offloading devices. ${ }^{17}$

TCCs are casts molded to the shape of the plantar foot that extend from the distal phalanges, across the osseous prominences of the plantar foot and beyond the back of the heel, proximally up the leg. They work by grossly distributing up the cast any forces to the foot, thereby shielding active wounds from direct, forceful trauma that would further initiate ulcerative development. ${ }^{18}$ TCCs can reduce pressure at the site of neuropathic ulcers by $84 \%-92 \%$ and have the ability to heal most diabetic neuropathic ulcers in 6-8 weeks. ${ }^{11}$ TCCs are also effective in controlling edema and the osseous changes seen with the initial phases of Charcot neuroarthropathy. ${ }^{19}$

As mentioned earlier, a majority of clinicians in 2008 study preferred not to use TCCs due to the learning curve and perceived cost-benefit analysis of using shoe modifications over casting. Figure 2 shows a TCC, the gold standard in offloading neuropathic wounds, which is quite cumbersome for patients and requires significant time and expertise to apply properly. ${ }^{8}$ Although effective in patients with moderate ischemia or infection, TCCs are strongly contraindicated in patients with significant peripheral arterial disease. ${ }^{19}$ When 


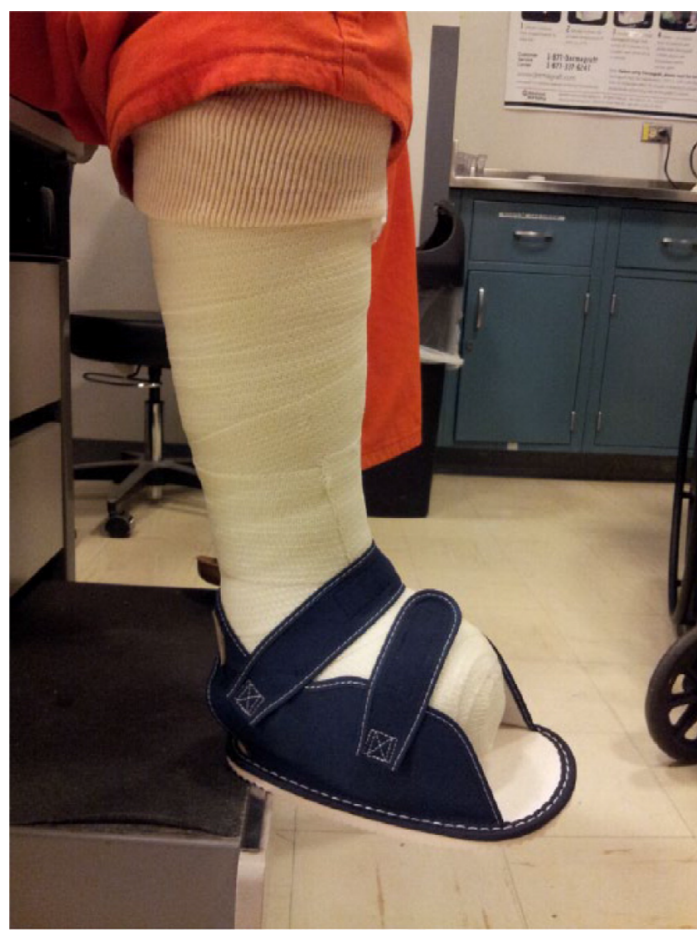

Figure 2 Total contact casts are the gold standard for offloading diabetic neuropathic wounds, yet are quite cumbersome for patients and take significant time and expertise to apply properly in the clinical setting.

applied haphazardly, it can worsen a wound and allow the spread of infection as the cast prevents daily wound inspections and dressing changes.

Removable cast walkers (RCWs) are one alternative to the TCC in that they are rapidly applied and equally efficacious, are easily removed to wash and inspect wounds, and allow patients to ambulate with adequate plantar offloading via the rocker bottom sole. RCWs are also an excellent alternative to TCCs for patients with vascular diseases like critical limb ischemia. ${ }^{20}$ The advantage of the easily applied and removed $\mathrm{RCW}$ is also its weakness in that patient adherence to the walker is a major factor in nonhealing ulcers and frequent recurrence in those who do not continually wear their RCWs.

A hybrid device known as the "instant TCC" utilizes the frame of the RCW alongside the semipermanent binding of TCCs. By wrapping the RCW with a cohesive bandage (Figure 3), patients benefit from the forced compliance of a TCC while enjoying the more tolerable offloading capability of an $\mathrm{RCW}^{21}$

\section{Surgical methods of offloading Achilles tendon lengthening}

Achilles tendon lengthening is an effective method to reduce the recurrence of plantar diabetic neuropathic ulcers of the forefoot in patients with limited ankle range of motion. The percutaneous procedure involves three stab incisions and can

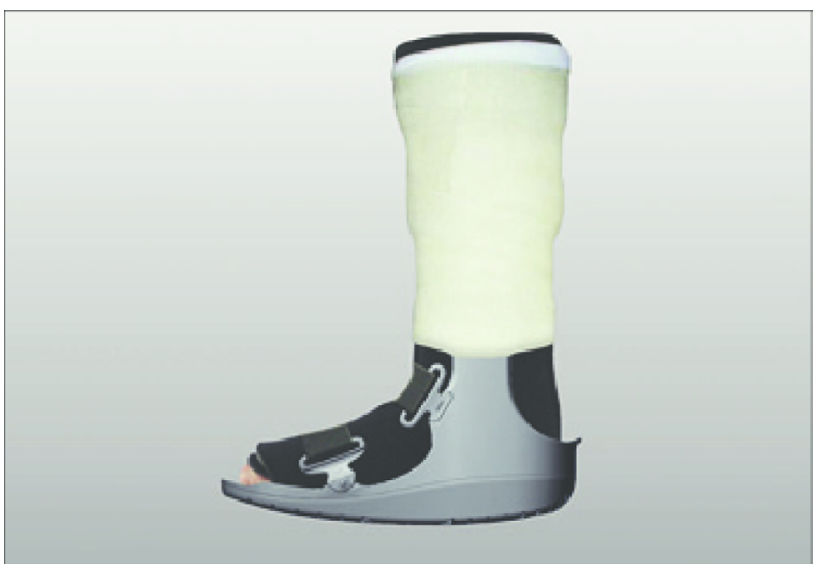

Figure 3 The instant total contact cast.

Note: A removable cast walker rendered irremovable due to wrapping of the upper portion with a layer of fiberglass.

be done with the patient lying prone under local anesthesia. ${ }^{22}$ A 1999 study showed a mean $28 \%$ reduction in peak pressures on the plantar aspect of the forefoot following percutaneous Achilles tendon lengthening in diabetic patients who are at high risk for ulcerations of the foot. ${ }^{23}$ A subsequent study in 2003 demonstrated that Achilles tendon lengthening procedures in conjunction with TCC application reduced the recurrence of ulceration from $59 \%$ to $15 \%$ compared with TCC applications alone. ${ }^{24}$

\section{Surgical correction of skeletal abnormalities}

Deformities of the foot's natural osseous architecture is highly linked to ulcer recurrence; therefore, surgical debridement of the abnormal bones is often necessary to aid in prolonging ulcerative remission. ${ }^{25}$ Over $50 \%$ of plantar DFUs are found under the hallux or first metatarsal head. If these skeletal abnormalities are flexible, tenotomies such as flexor tenotomies to offload the area of ulceration on the distal hallux may be preferred to osseous corrections. If the skeletal abnormalities are rigid with a digital ulceration, as seen with hallux valgus or limitus, osseous procedures like arthroplasties of the base of the proximal phalanx of the hallux (a Keller procedure) with reinsertion of the flexor hallucis brevis are recommended to prevent further ulceration. ${ }^{26}$ By correcting hammertoes of the lesser digits, pressure is mitigated on the distal tips and dorsal joint surfaces. If unsuccessful and ulceration persists plantar to the metatarsal head, single or pan metatarsal resection can facilitate healing. ${ }^{27}$

\section{Plantar fat pad augmentation}

Soft tissue that has healed after ulceration can remain with significant substance deficit and little tissue protecting the 
area. As plantar pressure is a direct result of plantar fat pad thickness, new efforts are being directed toward ameliorating this. Prior reports of silicone injection treatment to add bulk to soft tissue associated with ulceration or preulceration have thoroughly indicated its safe utility in prevention and maintenance of wound remission. ${ }^{28}$ In addition, current efforts are being made to optimize the use of one's own adipocytes to increase soft tissue depth and promote autogenous sourcing of graft material in preventing the recurrence of DFUs. ${ }^{29}$ Current reports demonstrate the efficacy in the injection of autologous abdominal fat into the areas of plantar prominence to reduce lesion recurrence or new ulceration formation. ${ }^{29}$

\section{Monitoring strategies and technological innovations Wearables}

The emergence of "smart" technologies and wearable electronics paves the way for the integration of both in the context of providing patients and clinicians with objective data about patient health that is easily accessible. Physicians no longer need to rely on the subjective history given by neuropathic patients who lack the ability to sense the deterioration of their own bodies. ${ }^{30}$ Such information can now be obtained with the advent of "smart" wearable technologies like SmartSox ${ }^{31}$ and other wearables to detect the effect of physical activity on the body (Figure 4). The success or failure of various treatment regimens instituted for diabetics with neuropathy might correlate with the levels of physical activity undertaken by the patient, as the neuropathic ulcers typically develop due to repetitive stress applied to the feet during weight-bearing. ${ }^{32}$

Because shear stresses contribute to the formation of diabetes-related foot ulcers, a 2014 study published in the Journal of Diabetes Science and Technology evaluated the effect of a custom, novel shear-reducing insole on the thermal

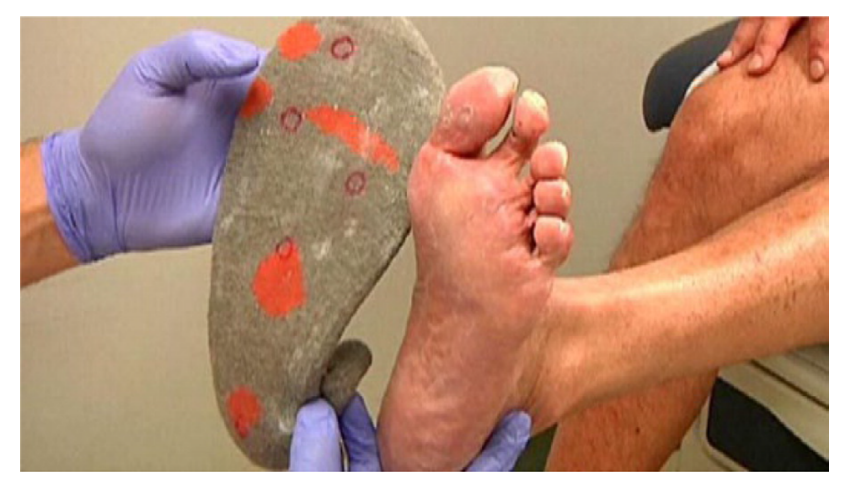

Figure 4 SmartSox uses fiber optic cables embedded within socks to warn the patient of peaks in plantar pressure.

Note: Warning messages are sent to the patient's wristwatch, instructing them to offload the areas of high pressure. response to ambulation and found significant reductions in forefoot and midfoot temperature increases after walking when using the customizable insole compared with standard insoles. ${ }^{33}$

As constant monitoring paves the way for patient-specific medicine, the implementation and proliferation of 3D printing to personalize exoskeleton suits has found its place in helping paraplegics walk, military personnel carry heavier loads in a more efficient manner, and benefit frail and highrisk diabetics with neuropathy reduce plantar tissue impact and reduce the stress of weight-bearing. ${ }^{34}$

These findings and similar research on customizable, wearable technologies merit future research on such devices for the prevention of neuropathic ulcers and for prolonging the remission of previous ulceration.

\section{At-home monitors}

A 2007 study found that those who did not use a system for temperature monitoring system quadrupled their chances of developing foot ulcer recurrence than those who did, emphasizing the major impact this technology could have on global health if implemented ubiquitously. ${ }^{35}$ Constant monitoring systems for diabetics will include such technologies that will be integrated into the daily life of the patients via wearable sensors and smart technologies such as "intelligent" insoles or "smart" socks, which can detect and alert both the patient and the physician about the need for possible intervention before ulceration can manifest itself as a neuropathic wound. Some of the earliest known clinical markers for the inflammatory process that occurs prior to ulceration include calor, erythema, and high peak plantar pressures. ${ }^{36}$ The technology behind the "smart" socks, for example, include intelligent textiles that use fiber optics and sensors to monitor pressure, temperature, and joint angles of the feet of neuropathic diabetics who cannot otherwise detect these sensations themselves, and simultaneously measure all three parameters to precisely indicate the location on the foot, which is likely to develop an ulcer if left untreated. ${ }^{31}$

\section{The role of the multidisciplinary team (MDT)}

A coordinated team approach has also been shown to decrease the frequency of limb loss in diabetic patients worldwide. ${ }^{37-39}$ In the Netherlands, and recently the USA, the inclusion of podiatrists in a multidisciplinary approach to diabetic foot disease has reduced amputations by $34 \%$ and $64 \%$, respectively. ${ }^{40,41}$ Asian health care teams report strong correlation between teamwork and multidisciplinary protocol 
in diabetic foot care in reducing the rates of amputation. ${ }^{42,43}$ In the UK, an organized MDT approach to the diabetic foot care has shown a sustained reduction in amputation rates. ${ }^{44,45}$ Italian literature also reports a reduction in hospitalization and major limb amputation, thanks to deployment of an MDT in high-risk patients. ${ }^{46}$ Although there is a significant amount of data to show that multidisciplinary protocol and teamwork reduce amputations, as yet there are no data to show definitively that a combination of advanced therapies and care via an MDT leads to shorter healing times. The benefits of an MDT are far reaching, including a focused approach to each patient's particular needs, an optimized multifaceted management of patients with DM, and an enhanced patient surveillance. ${ }^{47}$ When considering the makeup of an MDT, there is no established set of parameters. The International Diabetes Federation recommends that in order to provide comprehensive specialist foot care, an MDT should comprise doctors with a particular interest in diabetes, podiatrists, trained nurses, vascular surgeons, orthopedic surgeons, infection specialists, orthotists, social workers, and psychologists. However, despite the guidelines, local resources will govern the skill mix and scope of any foot care team. The key within any team is the ability to access immediately relevant health care professionals, for example, a vascular surgeon. In the UK, there have been moves toward establishing a core team of specialist diabetes podiatrists, medical specialty consultants, orthotists, and surgeons working with nurses and GPs in a "virtual" network. In many countries, there is a shortage of specialist practitioners, which makes establishing an MDT extremely difficult. However, nonspecialist practitioners can play a key role in the early detection of problems and prompt referrals. Ideally, one clinician should act as a coordinator (or gatekeeper) of multidisciplinary care to ensure that appropriate referrals are made and that care is integrated. The coordinator may be a podiatrist, a surgeon, or another type of clinician with a special interest in diabetes. In some countries, coordinated care may be based within a single clinic. ${ }^{47}$

\section{Lasting comments on ulcer remission}

In summary, we propose flipping the script, if you will, on our perspective on "wound healing". We propose focusing not on the staccato events that punctuate each acute on chronic event, but rather the entire score - from the beginning to the end. In this way, our perspective is enhanced, our therapies can be less reactive, and we may all find that we are playing together in the same symphony.

\section{Acknowledgment}

The patients provided written informed consent for their images to be used in this publication.

\section{Disclosure}

The authors report no conflicts of interest in this work.

\section{References}

1. Bloom DE, Cafiero E, Jané-Llopis E, et al. The global economic burden of noncommunicable diseases. Program on the Global Demography of Aging, 2012. Available from: https://ideas.repec.org/p/gdm/wpaper/8712.html. Accessed December 18, 2016.

2. American Diabetes Association. Standards of medical care in diabetes 2014. Diabetes Care. 2013;37:S14-S80.

3. Peters EJ, Armstrong DG, Lavery LA, Risk factors for recurrent diabetic foot ulcers: site matters. Diabetes Care. 2007;30(8):2077-2079.

4. Skrepnek GH, Mills JL, Lavery LA, Armstrong DG. Health Care Service and Outcomes Among an Estimated 6.7 Million Ambulatory Care Diabetic Foot Cases in the U.S. Diabetes Care. 2017. Epub 2017 May 11.

5. van Netten JJ, Price PE, Lavery LA, et al; International Working Group on the Diabetic Foot. Prevention of foot ulcers in the at-risk patient with diabetes: a systematic review. Diabetes Metab Res Rev. 2016;32(Suppl 1):84-98.

6. Armstrong DG, Boulton AJM, Bus S. Approach to Diabetic Foot Ulcers and Their Recurrence. New England Journal of Medicine. 2017;376(24): 2367-2375.

7. Armstrong DG, Mills JL. Toward a change in syntax in diabetic foot care: prevention equals remission. $J$ Am Podiatr Med Assoc. 2013;103(2):161-162.

8. Miller JD, Salloum M, Button A, Giovinco NA, Armstrong DG. How can I maintain my patient with diabetes and history of foot ulcer in remission? Int J Low Extrem Wounds. 2014;13(4):371-377.

9. Crews R. Diabetes: improving foot care compliance. Lower Extremity Rev Mag. 2009;1(4):25-30.

10. Armstrong DG, Boulton AJ. Activity monitors: should we begin dosing activity as we dose a drug? J Am Podiatr Med Assoc. 2001;91(3) $152-153$.

11. Cavanagh PR, Bus SA. Off-loading the diabetic foot for ulcer prevention and healing. Plast Reconstr Surg. 2011;127(Suppl 1):248S-256S.

12. Armstrong DG, Peters EJ, Athanasiou KA, Lavery LA. Is there a critical level of plantar foot pressure to identify patients at risk for neuropathic foot ulceration? J Foot Ankle Surg. 1998;37(4)303-307.

13. Najafi B, Grewal GS, Bharara M, Menzies R, Talal TK, Armstrong DG. Can't stand the pressure: the association between unprotected standing, walking, and wound healing in people with diabetes. J Diabetes Sci Technol. Epub 2016 Aug 10.

14. Wu SC, Jensen JL, Weber AK, Robinson DE, Armstrong DG. Use of pressure offloading devices in diabetic foot ulcers: do we practice what we preach? Diabetes Care. 2008;31(11):2118-2119.

15. Uccioli L, Faglia E, Monticone G, et al. Manufactured shoes in the prevention of diabetic foot ulcers. Diabetes Care. 1995;18(10):1376-1378

16. Bus SA, van Deursen RW, Armstrong DG, Lewis JE, Caravaggi C, Cavanagh PR. Footwear and offloading interventions to prevent and heal foot ulcers and reduce plantar pressure in patients with diabetes: a systematic review. Diabetes Metab Res Rev. 2016;32(Suppl 1):99-118.

17. Morona JK, Buckley ES, Jones S, Reddin EA, Merlin TL. Comparison of the clinical effectiveness of different off-loading devices for the treatment of neuropathic foot ulcers in patients with diabetes: a systematic review and meta-analysis. Diabetes Metab Res Rev. 2013;29(3):183-193.

18. Lavery LA, Baranoski S, Ayello EA. Options for off-loading the diabetic foot. Adv Skin Wound Care. 2004;17(4 Pt 1):181-182, 184-186.

19. Rogers LC, Frykberg RG, Armstrong DG, et al. The charcot foot in diabetes. Diabetes Care. 2011;34:2123-2129. 
20. Brem H, Sheehan P, Boulton AJ. Protocol for treatment of diabetic foot ulcers. Am J Surg. 2004;187(5A):1S-10S.

21. Armstrong DG, Short B, Espensen EH, Abu-Rumman PL, Nixon BP, Boulton AJ. Technique for fabrication of an "instant total-contact cast" for treatment of neuropathic diabetic foot ulcers. $J$ Am Podiatr Med Assoc. 2002;92(7):405-408.

22. Nishimoto GS, Attinger CE, Cooper PS. Lengthening the Achilles tendon for the treatment of diabetic plantar forefoot ulceration. Surg Clin North Am. 2003;83(3):707-726.

23. Armstrong DG, Stacpoole-Shea S, Nguyen H, Harkless LB. Lengthening of the Achilles tendon in diabetic patients who are at high risk for ulceration of the foot. J Bone Joint Surg Am. 1999;81(4):535-538.

24. Mueller MJ, Sinacore DR, Hastings MK, Strube MJ, Johnson JE. Effect of Achilles tendon lengthening on neuropathic plantar ulcers. A randomized clinical trial. J Bone Joint Surg Am. 2003;85-A(8):1436-1445.

25. Armstrong DG, Lavery LA, Stern S, Harkless LB. Is prophylactic diabetic foot surgery dangerous? J Foot Ankle Surg. 1996;35(6):585-589.

26. Armstrong DG, Frykberg RG. Classifying diabetic foot surgery: toward a rational definition. Diabet Med. 2003;20(4):329-331.

27. La Fontaine J, Lavery LA, Hunt, Murdoch DP. The role of surgical off-loading to prevent recurrent ulcerations. Int J Low Extrem Wounds. 2014;13(4):320-334.

28. Balkin SW. Injectable silicone and the foot: a 41-year clinical and histologic history. Dermatol Surg. 2005;31(11 Pt 2):1555-1559; discussion 1560.

29. Luu CA, Larson E, Rankin TM, Pappalardo JL, Slepian MJ, Armstrong DG. Plantar fat grafting and tendon balancing for the diabetic foot ulcer in remission. Plastic Reconstr Surg Glob Open. 2016;4(7):e810.

30. Ferber R, Webber T, Everett B, Groenland M. Validation of plantar pressure measurements for a novel in-shoe plantar sensory replacement unit. J Diabetes Sci Technol. 2013;7(5):1167-1175.

31. Najafi B. SmartSox: a smart textile to prevent diabetic foot amputation. Qatar Found Ann Res Forum Proce. 2013;BIOP 013

32. Najafi B, Armstrong DG, Mohler J. Novel wearable technology for assessing spontaneous daily physical activity and risk of falling in older adults with diabetes. J Diabetes Sci Technol. 2013:7(5):1147-1160.

33. Wrobel JS, Ammanath P, Le T, et al. A novel shear reduction insole effect on the thermal response to walking stress, balance, and gait. $J$ Diabetes Sci Technol. 2014;8(6):1151-1156.

34. Rankin TM, Giovinco NA, Cucher DJ, Watts G, Hurwitz B, Armstrong DG. Three-dimensional printing surgical instruments: are we there yet? J Surg Res. 2014;189(2):193-197.

35. Lavery LA, Higgins KR, Lanctot DR, et al. Preventing diabetic foot ulcer recurrence in high-risk patients: use of temperature monitoring as a self-assessment tool. Diabetes Care. 2007;30(1):14-20.
36. Bharara M, Schoess J, Armstrong DG. Coming events cast their shadows before: detecting inflammation in the acute diabetic foot and the foot in remission. Diabetes Metab Res Rev. 2012;28(Suppl 1):15-20.

37. Larsson J, Apelqvist J, Agardh CD, Stenström A. Decreasing incidence of major amputation in diabetic patients: a consequence of a multidisciplinary foot care team approach? Diabet Med. 1995;12(9):770-776.

38. Krishnan S, Nash F, Baker N, Fowler D, Rayman G. Reduction in diabetic amputations over 11 years in a defined UK population benefits of multidisciplinary team work and continuous prospective audit. Diabetes Care. 2008;31(1):99-101.

39. Tseng CL, Rajan M, Miller DR, Lafrance JP, Pogach L. Trends in initial lower extremity amputation rates among Veterans Health Administration health care system users from 2000 to 2004. Diabetes Care. 2011;34(5):1157-1163.

40. van Houtum WH, Rauwerda JA, Ruwaard D, Schaper NC, Bakker $\mathrm{K}$. Reduction in diabetes-related lower-extremity amputations in The Netherlands: 1991-2000. Diabetes Care. 2004;27(5):1042-1046.

41. Sloan FA, Feinglos MN, Grossman DS. Receipt of care and reduction of lower extremity amputations in a nationally representative sample of U.S. Elderly. Health Serv Res. 2010;45(6 Pt 1):1740-1762.

42. Aksoy DY, Gürlek A, Cetinkaya Y, et al. Change in the amputation profile in diabetic foot in a tertiary reference center: efficacy of team working. Exp Clin Endocrinol Diabetes. 2004;112(9):526-530.

43. Rerkasem K, Kosachunhanun N, Tongprasert S, et al. Reducing lower extremity amputations due to diabetes: the application of diabetic-foot protocol in Chiang Mai University Hospital. Int J Low Extrem Wounds. 2008;7(2):88-92.

44. Edmonds ME, Blundell MP, Morris ME, Maelor Thomas E, Cotton LT, Watkins PJ. Improved survival of the diabetic foot: the role of a specialised foot clinic. QJ Med. 1986;60(232):763-771.

45. Canavan RJ, Unwin NC, Kelly WF, Connolly VM. Diabetes-and nondiabetes-related lower extremity amputation incidence before and after the introduction of better organized diabetes foot care continuous longitudinal monitoring using a standard method. Diabetes Care. 2008;31(3):459-463.

46. Anichini R, Zecchini F, Cerretini I, et al. Improvement of diabetic foot care after the Implementation of the International Consensus on the Diabetic Foot (ICDF): results of a 5-year prospective study. Diabetes Res Clin Pract. 2007;75(2):153-158.

47. Sumpio BE, Armstrong DG, Lavery LA, Andros G. The role of interdisciplinary team approach in the management of the diabetic foot: a joint statement from the Society for Vascular Surgery and the American Podiatric Medical Association. J Am Podiatr Med Assoc. 2010;100(4):309-311.
Chronic Wound Care Management and Research

\section{Publish your work in this journal}

Chronic Wound Care Management and Research is an international, peer reviewed, open access, online journal publishing original research, reviews, editorials, and commentaries on the causes and management of chronic wounds and the major issues related to chronic wound management. Topics also include chronic wounds as comorbidities to other

\section{Dovepress}

conditions, patient adherence to therapy, and the economic burden of chronic wounds. The manuscript management system is completely online and includes a very quick and fair peer review system, which is all easy to use. Visit http://www.dovepress.com/testimonials.php to read real quotes from published authors. 